\title{
Next Generation QTAIM for the Design of Quinone-based Switches
}

\author{
Tian Tian ${ }^{1}$, Tianlv Xü ${ }^{1}$, Tanja van Mourik $^{2}$, Herbert Früchtl², Steven R. Kirk ${ }^{* 1}$ \\ and Samantha Jenkins ${ }^{* 1}$
}

${ }^{1}$ Key Laboratory of Chemical Biology and Traditional Chinese Medicine Research and Key Laboratory of Resource Fine-Processing and Advanced Materials of Hunan Province of MOE, College of Chemistry and Chemical Engineering, Hunan Normal University, Changsha, Hunan 410081, China

${ }^{2}$ EaStCHEM School of Chemistry, University of St Andrews, North Haugh, St Andrews, Fife KY16 9ST, Scotland, United Kingdom.

*email: samanthajsuman@gmail.com

*email: steven.kirk@cantab.net

Investigation of the hydrogen transfer tautomerization process yielded metallic hydrogen bonds in the benzoquinonelike core of the switch. Bond-path framework sets $B$ and $B_{\sigma}$, comprising a three-stranded, non-minimal 3-D bond, which included the familiar QTAIM bond-path and two additional paths defining the least and most preferred directions of electron density motion, were used with QTAIM and the stress-tensor respectively. The B and $\mathrm{B}_{\sigma}$ were visualized and uncovered the destabilizing effects on the hydrogen bond of the presence of an Fe atom. The lengths of $B$ and $B_{\sigma}$ quantified this effect and the dependence on the position of a fluorine substituent. 
The goal of achieving easier synthesis of molecules with the desired switching properties than the model system proposed in $\operatorname{Ref}^{1}$ requires a better understanding of electronic structure. In particular, this would allow prediction of the effect of the location and type of side groups attached to the quinone core, thus enabling finetuning of switching barrier and conductivity. Recent reviews where breakthroughs in electronic components and molecular junctions have recently been reported include those by Zhang et $a l^{2}$ and Komoto et $a l^{3}$, but a practical strategy has yet to be discovered.

Recently developed "next-generation" QTAIM (quantum theory of atoms in molecules) ${ }^{9}$ has provided a set of tools to study the bond structure of molecular systems ${ }^{4-7}$, which can be used to improve our understanding of molecular switches. A recent investigation by some of the current authors ${ }^{8}$ focussed on 3-imino-6methylenecyclohexa-1,4-diene-1,4-diamine that we referred to as the quinone switch, with F substituted in two locations (UP or DOWN) for the ring hydrogens and with and without a coordinated Fe atom above the ring, see Scheme 1. The UP and DOWN labels correspond to the F being bound to C3 or C6, respectively. Scheme 1 shows molecular graphs with F in the UP position. The operation of the switch consists in the movement of $\mathrm{H} 13$ from being bonded to N10 to being bonded to N11.
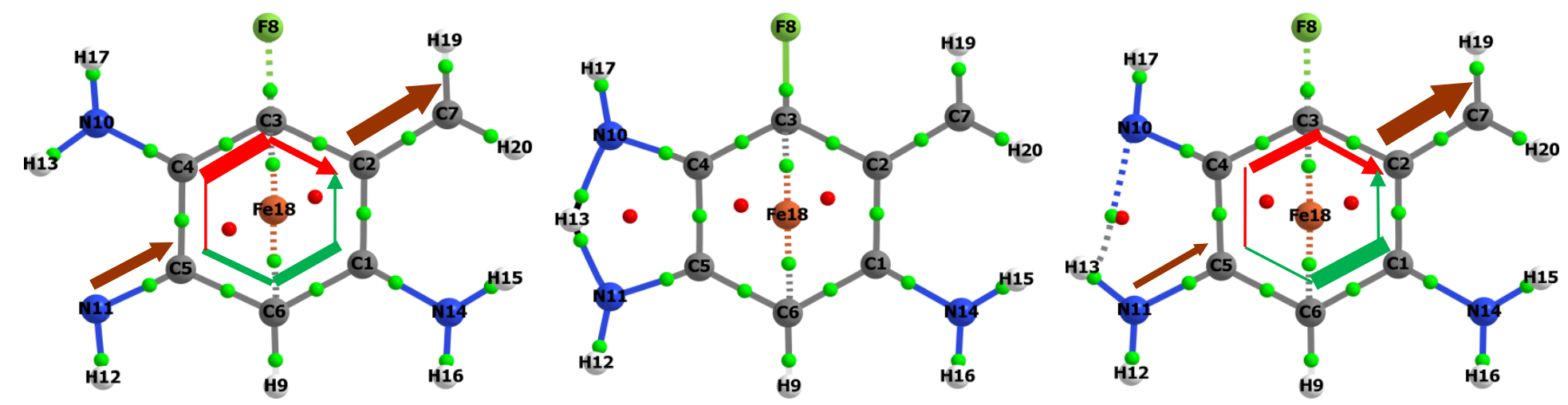

(a)
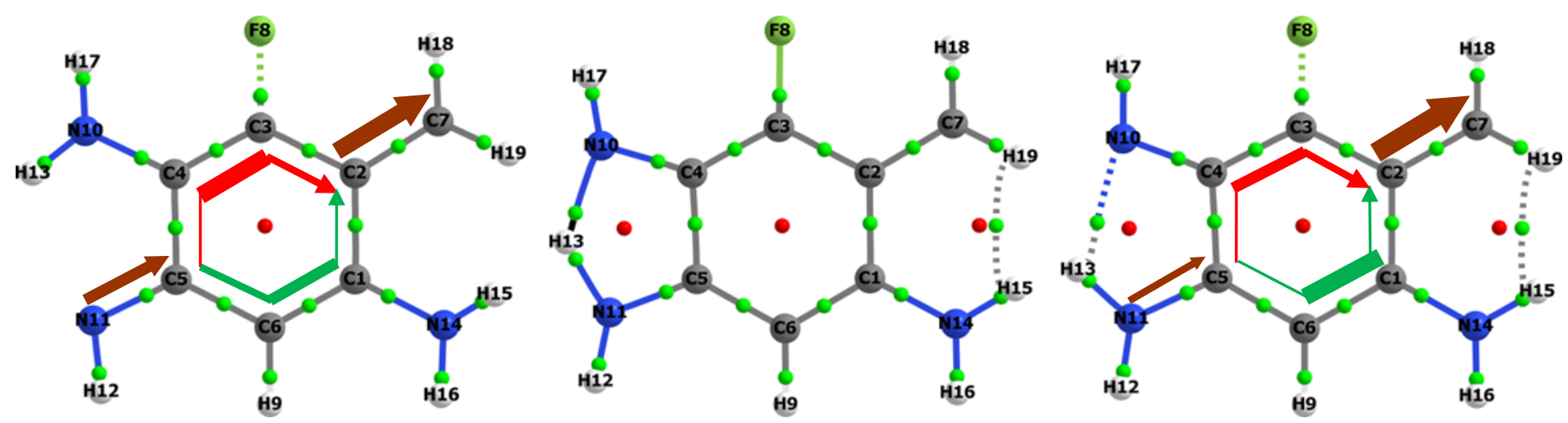

(b)

Scheme 1. Snapshots of the molecular graphs of the F-decorated quinone switch is shown doped with Fe in sub-figure (a) and undoped in sub-figure (b). The left (the reverse minimum $(r)$ ) and right panel (forward minimum $(f)$ ) correspond to the 'ON' position and 'OFF' positions of the switch, respectively, with the middle panel corresponding to the transitions state (TS) of the intrinsic reaction coordinate (IRC). The thickness of the red, green and brown arrows represent the magnitudes of the ellipticity $\varepsilon$ of the North pathway (C5-C4-C3-C2), South pathway (C5-C6-C1-C2), and the N11-C5 $B C P$ ('entry' pathway) and C2-C7 BCP ('exit' pathway), respectively ${ }^{8}$. 
This demonstrated that a single coordinated Fe atom significantly lowered the energy difference and barrier height, similar to the effect of a metal surface. As a consequence the coordinated Fe atom was found to greatly improve the switching properties, both in terms of the tautomerization barrier and the expected conductance behavior, while the effects of the position of the F-substitution (UP or DOWN) were found to be more subtle, see Scheme 2.

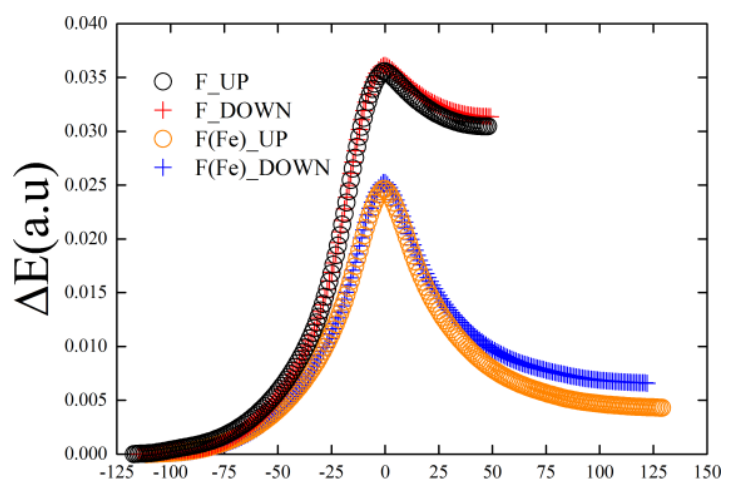

IRC-Step

Scheme 2. The relative energy $\triangle \mathrm{E}$ of the $\mathrm{F}(\mathrm{Fe}) \mathrm{UP}$ and $\mathrm{F}(\mathrm{Fe}) \mathrm{DOWN}$ substituted reaction pathways with coordinated Fe atom NCP are indicated by the black circles and red pluses respectively. The corresponding $\triangle \mathrm{E}$ plots without Fe (F UP and F DOWN) are indicated by the orange circles and blue pluses respectively. See Scheme 1 for the positions of the F (UP) and F (DOWN) locations respectively.

Previously, QTAIM ${ }^{9}$ and a non-Cartesian stress tensor phase space were constructed to determine the functioning of the switch in terms of the most and least preferred directions of electronic charge density accumulation $^{8}$. This was undertaken using the scalar ${ }^{4-6}$ and vector-based ${ }^{5,7}$ aspects the and the stress tensor formalism at the bond critical point $(B C P)$.

In this investigation we will use the recently introduced 3-D bond-path framework set $\mathrm{B}^{10}$ with both QTAIM and the stress tensor $\left(B_{\sigma}\right)$ that considers a non-minimal version of a bond that comprises three strands and follows changes along the entire length of a bond in terms of the most preferred and least preferred directions of electronic charge density motion.

We use QTAIM ${ }^{9}$ and the stress tensor analysis that utilizes higher derivatives of $\rho\left(\mathbf{r}_{\mathbf{b}}\right)$, in effect acting as a 'magnifying lens' on the $\rho\left(\mathbf{r}_{\mathbf{b}}\right)$ derived properties of the wave-function. QTAIM allows us to identify critical points in the total electronic charge density distribution $\rho(\mathbf{r})$ by analyzing the gradient vector field $\nabla \rho(\mathbf{r})$. These critical points can be divided into four types of topologically stable critical points according to the set of ordered eigenvalues $\lambda_{1}<\lambda_{2}<\lambda_{3}$, with corresponding eigenvectors $\underline{\mathbf{e}}_{1}, \underline{\mathbf{e}} \mathbf{2}$, $\underline{\mathbf{e}}_{3}$ of the Hessian matrix. In the limit that the forces on the nuclei become vanishingly small, an atomic interaction line (AIL) ${ }^{11}$ becomes a bondpath, although not necessarily a chemical bond ${ }^{12}$. The complete set of critical points together with the bondpaths of a molecule or cluster is referred to as the molecular graph, with the constituent atoms being referred to as nuclear critical points $(N C P \mathrm{~s})$.

The ellipticity $\varepsilon$ provides the relative accumulation of $\rho\left(\mathbf{r}_{\mathbf{b}}\right)$ in the two directions perpendicular to the bondpath at a $B C P$, defined as $\varepsilon=\left|\lambda_{1}\right| /\left|\lambda_{2}\right|-1$ where $\lambda_{1}$ and $\lambda_{2}$ are negative eigenvalues of the corresponding $\underline{\mathbf{e}} \mathbf{1}$ and 
$\underline{\mathbf{e}}_{2}$ respectively. It has been shown ${ }^{13,14}$ that the degree of covalent character can be determined from the total local energy density $H\left(\mathbf{r}_{\mathrm{b}}\right)$, defined as:

$$
H\left(\mathbf{r}_{\mathrm{b}}\right)=G\left(\mathbf{r}_{\mathrm{b}}\right)+V\left(\mathbf{r}_{\mathrm{b}}\right)
$$

In equation (1), $G\left(\mathbf{r}_{\mathrm{b}}\right)$ and $V\left(\mathbf{r}_{\mathrm{b}}\right)$ are the local kinetic and potential energy densities at a $B C P$, respectively. A value of $H\left(\mathbf{r}_{\mathrm{b}}\right)<0$ for the closed-shell interaction, $\nabla^{2} \rho\left(\mathbf{r}_{\mathrm{b}}\right)>0$, indicates a $B C P$ with a degree of covalent character, and conversely $H\left(\mathbf{r}_{\mathrm{b}}\right)>0$ reveals a lack of covalent character for the closed-shell $B C P$. Throughout this work, we use the terminology '--' and '---' to refer to closed-shell $B C P$ s which by definition always possess values of the Laplacian $\nabla^{2} \rho\left(\mathbf{r}_{\mathrm{b}}\right)>0$ but possess $H\left(\mathbf{r}_{\mathrm{b}}\right)<0$ or $H\left(\mathbf{r}_{\mathrm{b}}\right)>0$ respectively. Examples include the N10--H13/N10---H13 BCP, see Scheme 1. Conversely '-' always refers to shared-shell $B C P$ s which by definition always possess values of the Laplacian $\nabla^{2} \rho\left(\mathbf{r}_{\mathrm{b}}\right)<0$ and $H\left(\mathbf{r}_{\mathrm{b}}\right)<0$, e.g. the double bond C2-C7 BCP, see Scheme 1.A related quantity to the ellipticity $\varepsilon$ for closed-shell interactions is the metallicity:

$$
\xi\left(\mathbf{r}_{\mathrm{b}}\right)=\rho\left(\mathbf{r}_{\mathrm{b}}\right) / \nabla^{2} \rho\left(\mathbf{r}_{\mathrm{b}}\right) \geq 1,
$$

In equation (2) the values of total electronic charge density $\rho\left(\mathbf{r}_{\mathrm{b}}\right)$ and the Laplacian $\nabla^{2} \rho\left(\mathbf{r}_{\mathrm{b}}\right)$ respectively are calculated at the $B C P$. The metallicity $\xi\left(\mathbf{r}_{\mathrm{b}}\right)^{15,16}$ previously has been used to explore suspected metallicity ranges of metals, metalloids and non-metals ${ }^{15,16}$. Some of the current authors also demonstrated that the $\xi\left(\mathbf{r}_{\mathrm{b}}\right)$ is inversely related to "nearsightedness" of the first-order density matrix and is suitable for closed-shell systems $^{4}$. A set of results for the metallicity $\xi\left(\mathbf{r}_{\mathrm{b}}\right)$ of the N-H $B C P \mathrm{~s}$ for the complete intrinsic reaction coordinate (IRC) is presented in the Supplementary Materials S1. We will use the metallicity $\xi\left(\mathbf{r}_{\mathrm{b}}\right)$, that quantifies a relative delocalization of $\rho\left(\mathbf{r}_{\mathrm{b}}\right)$, to compare the facile character of the $\mathrm{H}$ atom participating in the tautomerization process, in the presence/absence of the Fe-dopant and UP/DOWN position of the Fsubstituent.

In this investigation we use bond-path framework set, $\mathrm{B}$, where $\mathrm{B}=\{\boldsymbol{p}, \boldsymbol{q}, \boldsymbol{r}\}$, the recently introduced nextgeneration QTAIM 3-D interpretation of the chemical bond. In the most general case a bond is comprised of three 'linkages'; $\boldsymbol{p}, \boldsymbol{q}$ and $\boldsymbol{r}$ associated with the $\underline{\mathbf{e}}_{\mathbf{1}}, \underline{\mathbf{e}}_{\mathbf{2}}$ and $\underline{\mathbf{e}}_{\mathbf{3}}$ eigenvectors, respectively. The $\boldsymbol{p}$ and $\boldsymbol{q}$ parameters define eigenvector-following paths with lengths $\mathrm{H}^{*}$ and $\mathrm{H}$, see Supplementary Materials S2.

$$
\begin{aligned}
& \mathbb{H}^{*}=\sum_{i=1}^{n-1}\left|\boldsymbol{p}_{i+1}-\boldsymbol{p}_{i}\right| \\
& \mathbb{H}=\sum_{i=1}^{n-1}\left|\boldsymbol{q}_{i+1}-\boldsymbol{q}_{i}\right|
\end{aligned}
$$


The lengths of the eigenvector-following paths $\mathrm{H}^{*}$ or $\mathrm{H}$ in equation (3a) and (3b) refer to the fact that the tips of the scaled $\underline{\mathbf{e}} \mathbf{1}$ or $\underline{\mathbf{e}} \mathbf{2}$ eigenvectors will sweep out along the extent of the bond-path, defined by the $\underline{\mathbf{e}} 3$ eigenvector, between the two bonded nuclei that the bond-path connects. In the limit of vanishing ellipticity $\varepsilon$ $=0$, for all steps $i$ along the bond-path length (BPL), one has $\mathrm{H}=$ BPL. For complete discussion and explanations refer to the Supplementary Materials S2. Two paths ( $\boldsymbol{q}$ and $\boldsymbol{q}$ ') are associated with the $\underline{\mathbf{e}}_{2}$ eigenvector since $\underline{\mathbf{e}}_{\mathbf{2}} \equiv \underline{\mathbf{e}}_{\mathbf{2}}$ lies in the same plane for the same point on the bond-path (r); correspondingly there are two paths associated with the $\underline{\mathbf{e}} \mathbf{1}\left(\boldsymbol{p}\right.$ and $\left.\boldsymbol{p}^{\prime}\right)$. The packet shapes that the pair of $\boldsymbol{q}$ - and $\boldsymbol{q}$ '-paths form along the $B C P$ will be referred to as a $\left\{\boldsymbol{q}, \boldsymbol{q}^{\prime}\right\}$ path-packet. The size, orientation and location of the $\{\boldsymbol{q}, \boldsymbol{q}$ ' $\}$ pathpackets indicate how much, which direction and whether a bond-path is twisted or linear. For instance, if a single long $\left\{\boldsymbol{q}, \boldsymbol{q}^{\prime}\right\}$-path packet occurs along the bond-path $(\boldsymbol{r})$ e.g. associated with the $\mathrm{H}-\mathrm{-N} B C P$, and $\mathrm{H}---\mathrm{H}$ $B C P$, that envelopes the $B C P$ then this is likely to indicate an easier (sliding) motion of the $B C P$, bond-path and associated $N C P$ s than for the twisted bond-path $(\boldsymbol{r})$ or a $\left\{\boldsymbol{q}, \boldsymbol{q}^{\boldsymbol{\prime}}\right\}$-path packet localized to the $B C P$. Longer $\{\boldsymbol{q}, \boldsymbol{q}\}$-paths, correspond to a greater tendency for motion along $\underline{\mathbf{e}} 2$ direction which corresponds to the easiest (most facile direction), see the Supplementary Materials S2. Larger $\left\{\boldsymbol{q}, \boldsymbol{q}^{\prime}\right\}$ and the stress tensor $\left\{\boldsymbol{q}_{\sigma}, \boldsymbol{q}_{\sigma}{ }_{\sigma}\right\}-$ path packets would therefore signify an easier passage of the $B C P$ and associated $\mathrm{H} N C P$. Note that the stress tensor $\left\{\boldsymbol{q}_{\sigma}, \boldsymbol{q}_{\sigma}\right\}^{\prime}$-path packets are constructed using the stress tensor ellipticity $\varepsilon_{\sigma}=\left|\lambda_{2 \sigma}\right| /\left|\lambda_{1 \sigma}\right|-1$.

Candidate structures for transition states were optimized with Gaussian $09^{17}$ using DFT at the PBE0 ${ }^{18} / \mathrm{cc}-$ pVTZ $^{19}$ level of theory, with Grimme's empirical 3-center dispersion correction with Becke-Johnson damping. ${ }^{20,21}$ Gaussian's 'ultrafine' DFT integration grid was used for all calculations. These settings were retained in all subsequent calculations described below. The presence of exactly one imaginary vibrational frequency was confirmed for each transition state structure. All IRC calculations were performed with a step size of 0.03 Bohr, tight SCF convergence criteria and a termination criterion of energy gradient magnitude $<$ $2 \times 10^{-4}$ Hartree/Bohr. The final calculated structures on each IRC path were then used as starting points for standard geometry optimizations, using the same method, basis set and DFT settings, to obtain precise local energy minimum structures to complete each full IRC path. For each generated structure representing a point on each computed IRC path, single-point SCF calculations were performed with the aforementioned DFT settings and basis set, with additional stricter convergence criteria; $<10^{-10}$ RMS change in the density matrix and $<10^{-8}$ maximum change in the density matrix. These calculations yielded the wave-functions needed for QTAIM analysis. Calculations of the molecular graphs and critical point properties were performed using AIMAll $^{22}$ : all molecular graphs were additionally confirmed to be free of non-nuclear attractor critical points. 


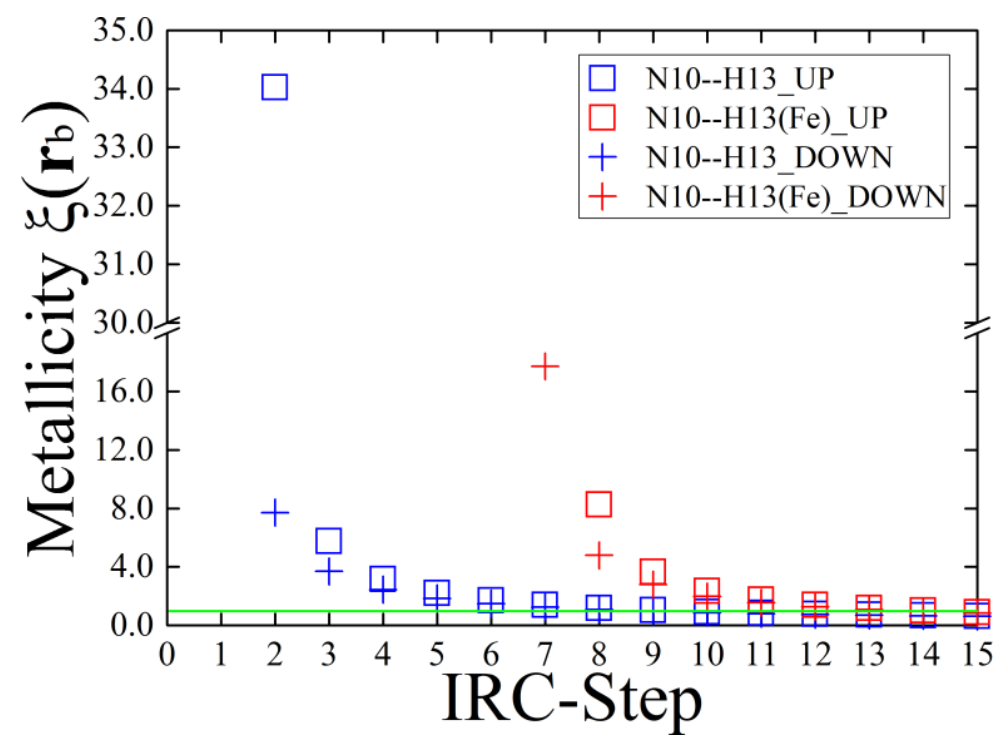

Figure 1. The variation of the metallicity $\xi\left(\mathbf{r}_{\mathrm{b}}\right)$ with the IRC from the transition state at IRC-Step $=0$ towards the forward minimum for N10--H13 BCP of the F-decorated quinone switch in the UP and DOWN positions for the undoped and Fe-doped molecular graph, see Scheme 1. The horizontal green line indicates values of metallicity $\xi\left(\mathbf{r}_{\mathrm{b}}\right)=1.0$.

The progress of the $\mathrm{H} \mathrm{NCP}$ away from the transition state during the tautomerization process leads to the creation of the metallic N10--H13 BCP, indicated by the presence of values of the metallicity $\xi\left(\mathbf{r}_{\mathrm{b}}\right) \geq 1$, see Figure 1 and the Supplementary Materials S1. The addition of the Fe atom leads to significantly different metallicity $\xi\left(\mathbf{r}_{b}\right)$ values where the difference between the values for the UP and DOWN positions of the Fsubstituent increases more with increase in the metallicity $\xi\left(\mathbf{r}_{\mathrm{b}}\right)$. The Fe-doped switch maintains higher $\xi\left(\mathbf{r}_{\mathrm{b}}\right)$ values further away from the transition state than the undoped switch, i.e. the $\xi\left(\mathbf{r}_{b}\right)$ values decrease much faster away from the transition state than the Fe-doped switch, see Figure 1. The rate of decrease of $\xi\left(\mathbf{r}_{\mathrm{b}}\right)$ values is: undoped $($ DOWN $)>$ undoped $($ UP $)>$ Fe-doped $($ DOWN $)>$ Fe-doped (UP), in the same order as the increase in the stabilities determined by the relative energy $\Delta \mathrm{E}$ for the forward direction, see Scheme 2.

Examination of the $\{\boldsymbol{q}, \boldsymbol{q}$ ' $\}$ path-packets for the shared-shell $B C P$ s demonstrates a low preference for the movement of the electronic charge density motion in the plane of the quinone switch, see Figure 2. The converse is true for the $\{\boldsymbol{p}, \boldsymbol{p}$ ' $\}$ path-packets of the shared-shell $B C P$ s, see Figure 3. The $\{\boldsymbol{q}, \boldsymbol{q}$ ' $\}$ and $\left\{\boldsymbol{p}, \boldsymbol{p}^{\text {' }}\right\}$ path-packets are so long for the Fe18--C3 $B C P$ and Fe18--C6 $B C P$, that for clarity they have been removed but the complete sets of paths are provided in the Supplementary Materials S3-S4. The undoped switch possesses very long unstable $\{\boldsymbol{q}, \boldsymbol{q}\}$ path-packets associated with the $\mathrm{H}---\mathrm{H} B C P$ bond-path for the transition state and forward minimum. The undoped switch with the F-substituent in the DOWN position is more topologically unstable than the UP position on the basis of having longer and even discontinuous $\{\boldsymbol{q}, \boldsymbol{q}\}$ pathpackets corresponding to the H---H $B C P$ bond-paths, see Figure 2(d) and Figure 2(b) respectively. The presence of the Fe atom destabilizes the N10--H13 $B C P$, as evidenced by the closer $B C P-R C P$ proximity compared with the undoped switch. This results in the $\left\{\boldsymbol{q}, \boldsymbol{q}^{\prime}\right\}$ and $\left\{\boldsymbol{q}_{\sigma}, \boldsymbol{q}^{\prime}{ }_{\sigma}\right\}$ path-packets for the Fe-doped molecule, displaying much larger extents in the vicinity of the N10--H13 BCP than for the undoped switch, e.g. compare the N10--H13 BCP in Figure 2(a-b) and Figure 4(a-b) respectively. The $\left\{\boldsymbol{p}_{\sigma, \boldsymbol{p}} \boldsymbol{p}_{\sigma}\right\}$ path-packets show the effect of the presence or absence of the Fe atom in terms of the least preferred directions of electronic charge density accumulation, see Figure 5. 

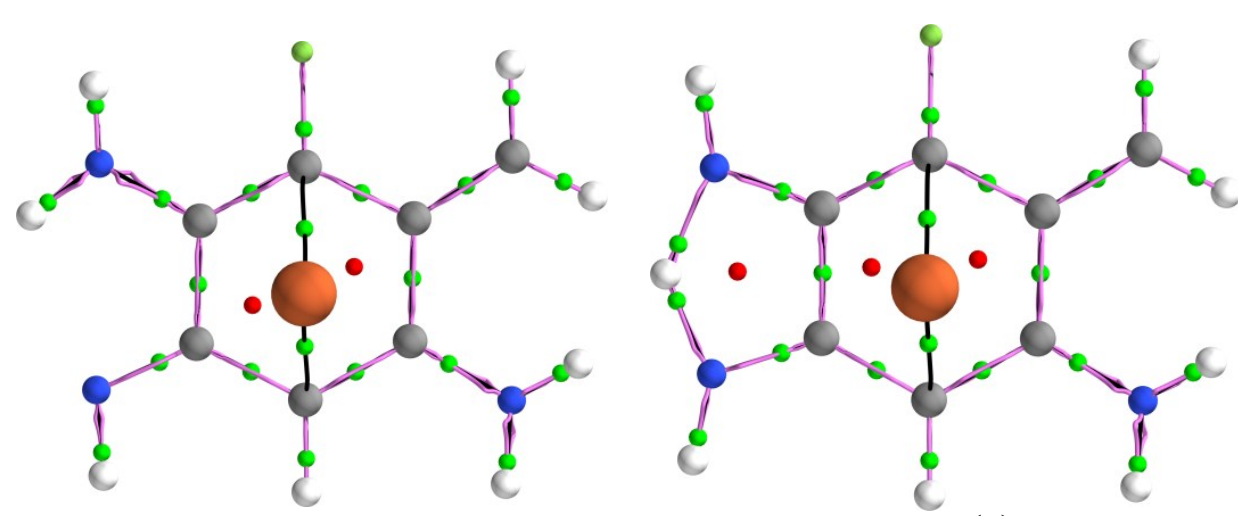

(a)
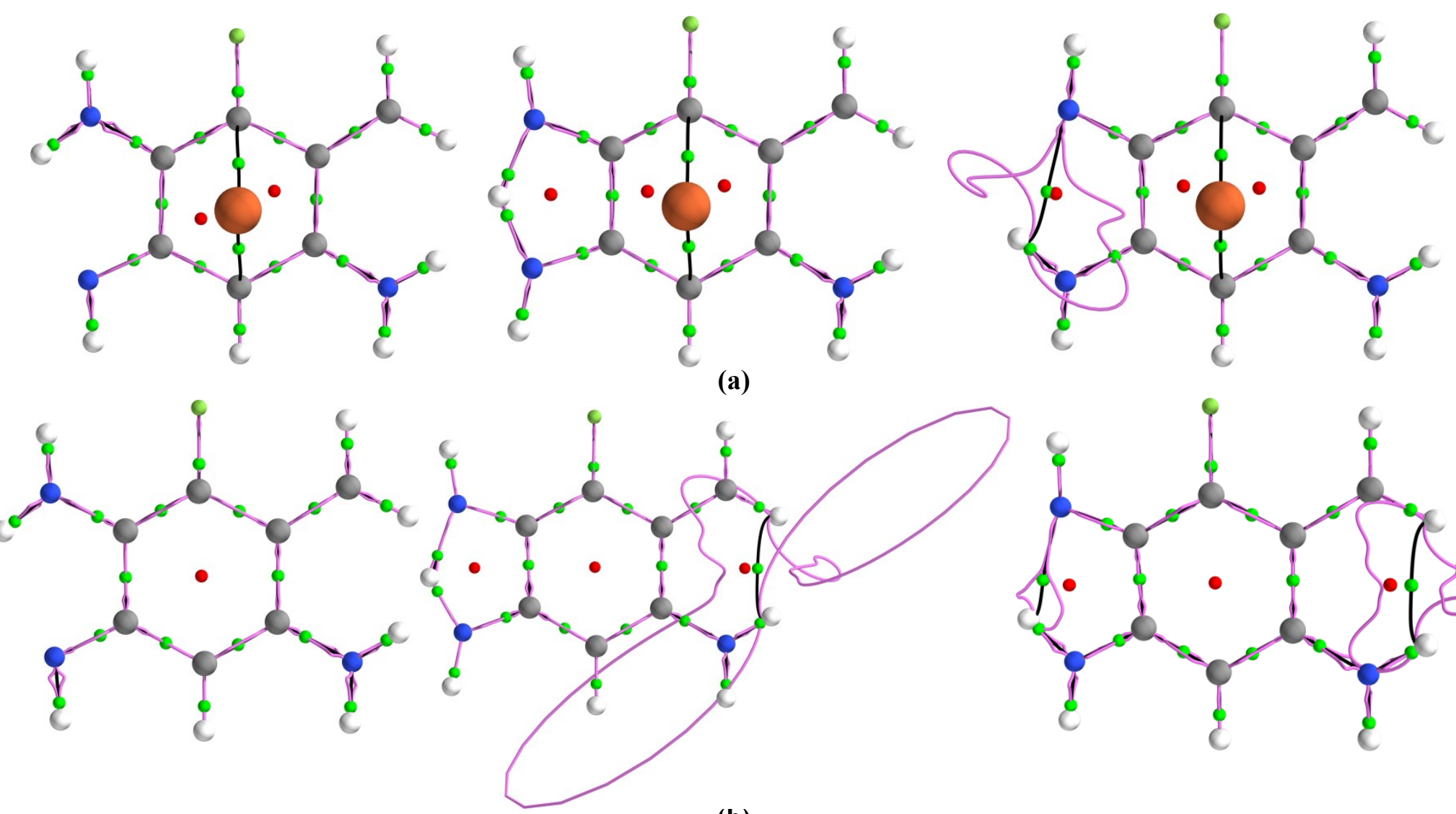

(b)
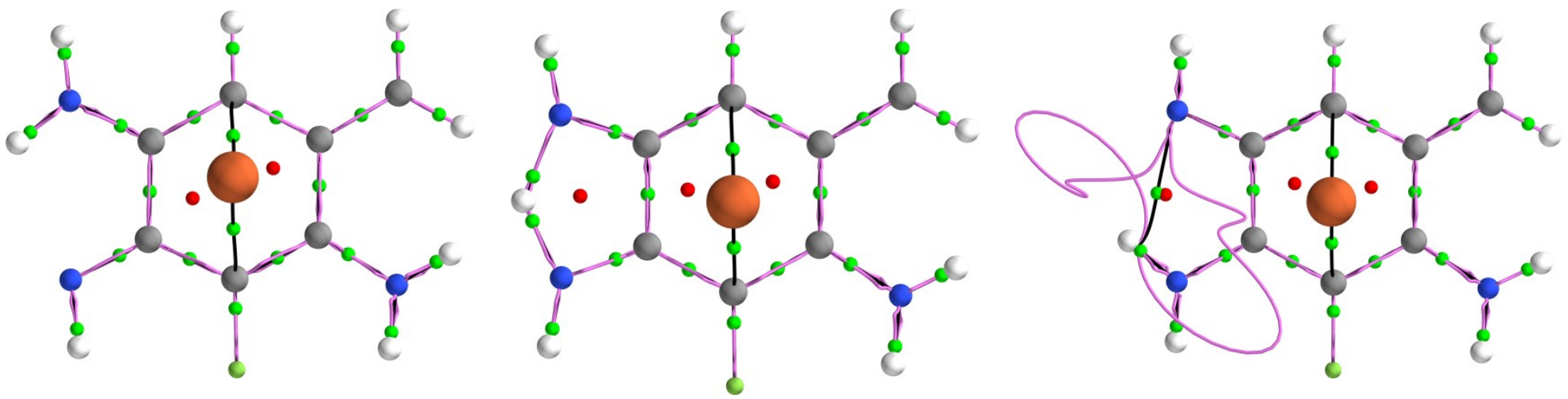

(c)
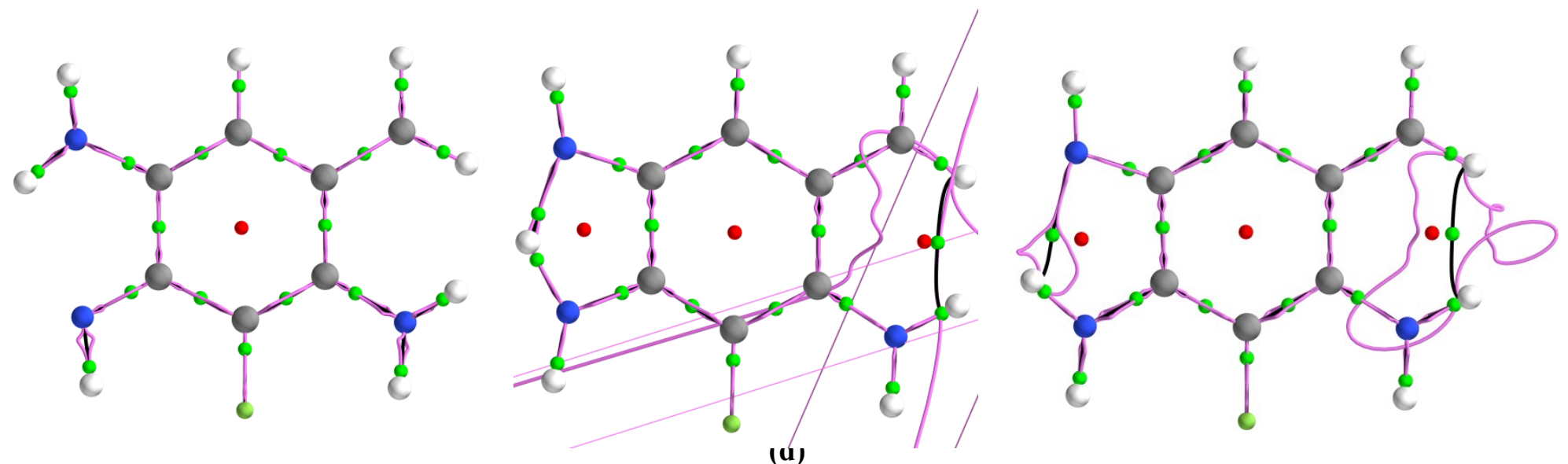

Figure 2. The $\left\{\boldsymbol{q}, \boldsymbol{q}^{\prime}\right\}$ path-packets of the Fe-doped and undoped quinone switch with F(UP) decoration presented in sub-figures (a-b) respectively and F(DOWN) in sub-figures (c-d) respectively. The undecorated green and red spheres indicate the positions of the bond critical points $(B C P \mathrm{~S})$ and ring critical points $(R C P)$ respectively, see Scheme $\mathbf{1}$ for the atom numbering scheme. Note, the extremely long $\left\{\boldsymbol{q}, \boldsymbol{q}^{\prime}\right\}$ path-packets associated with the Fe-C BCPs in sub-figures (a) and (c) have been omitted for clarity here but are provided in the Supplementary Materials S3. 

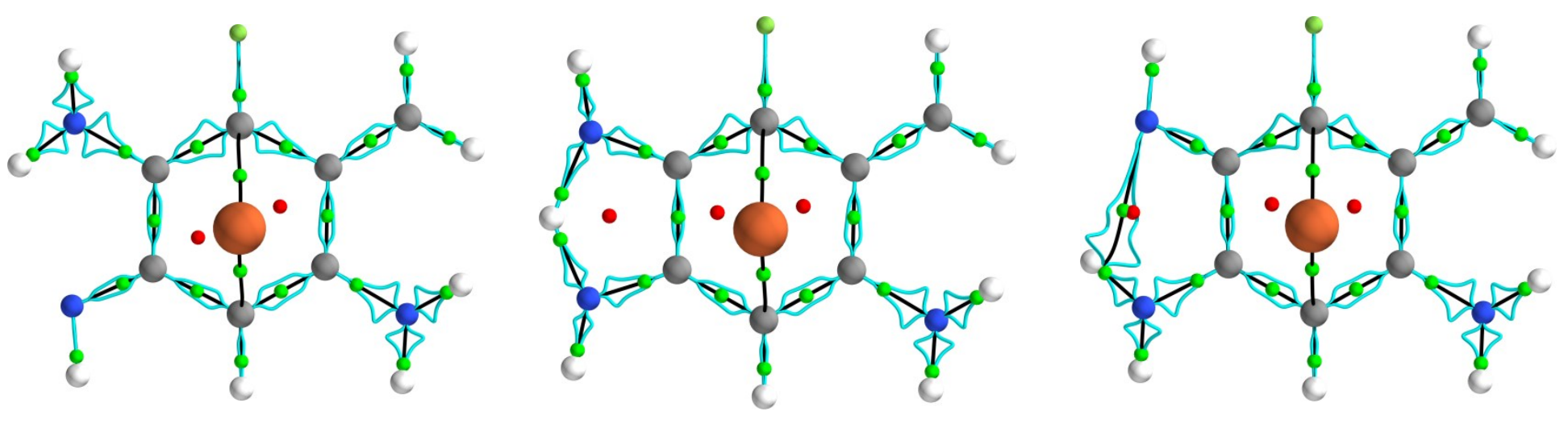

(a)
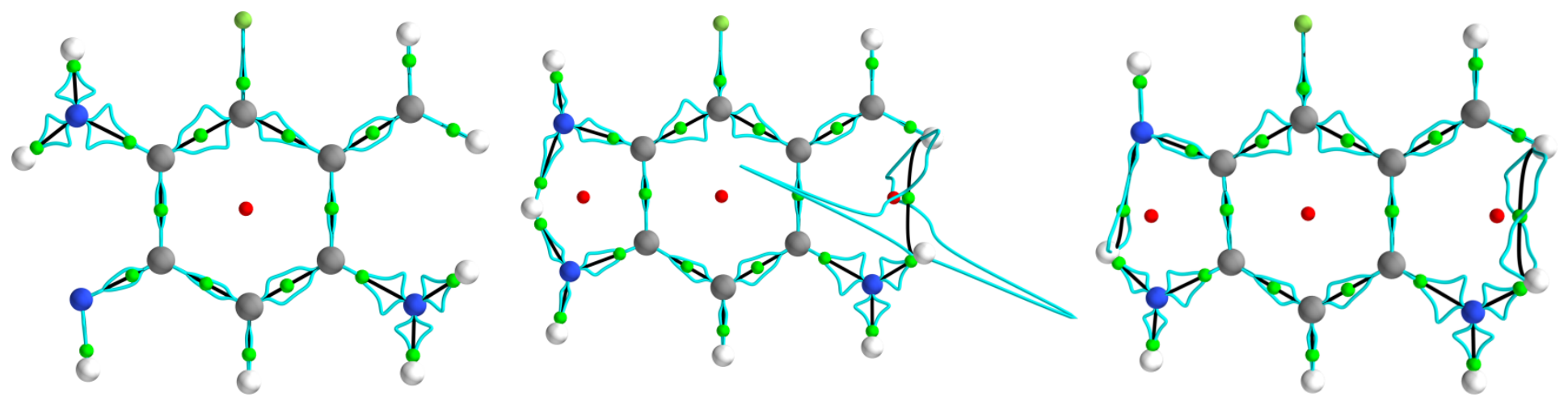

(b)
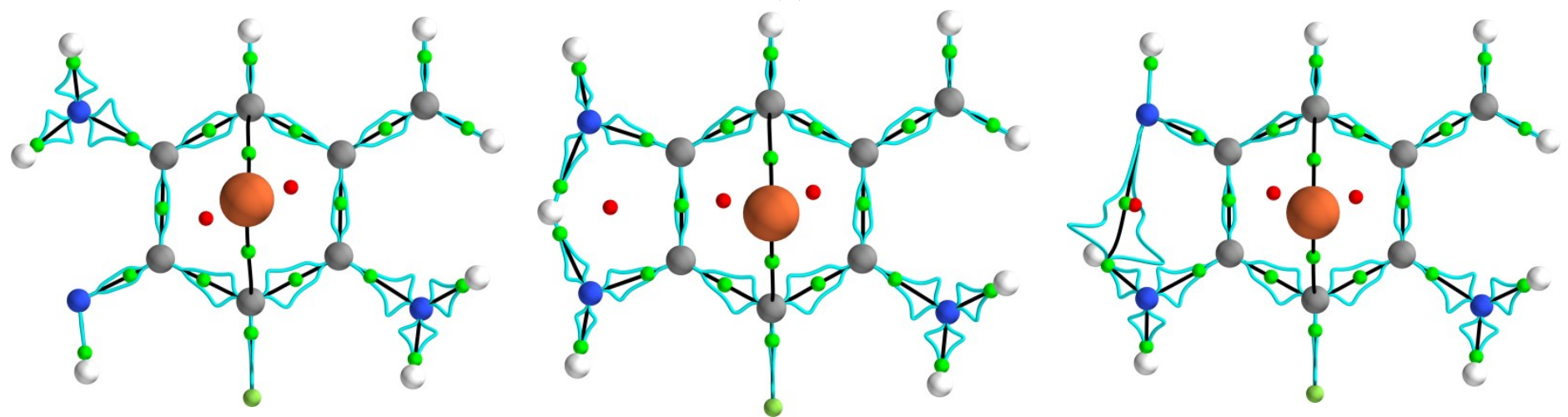

(c)
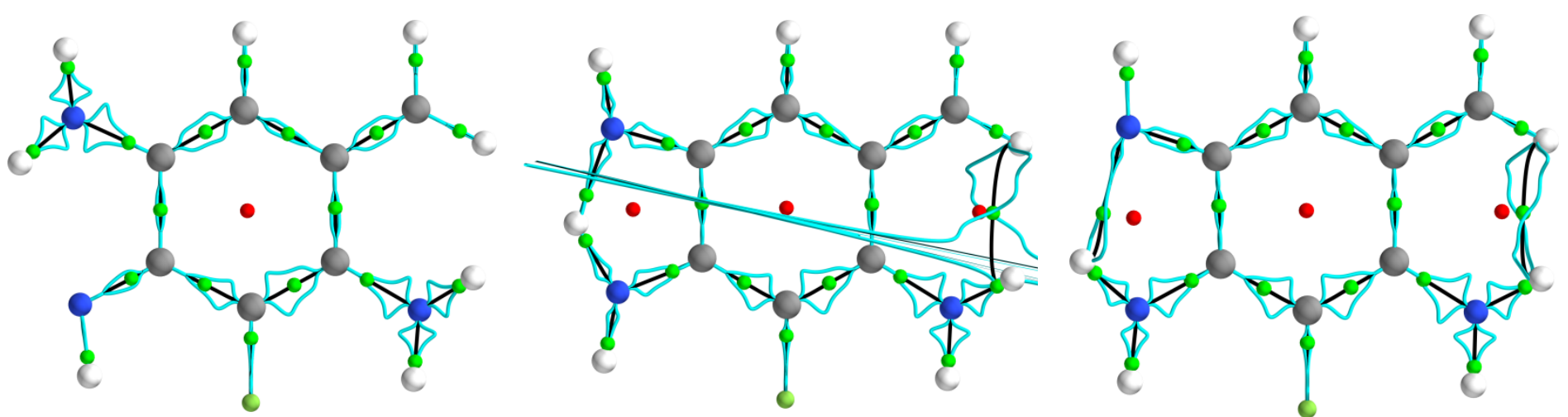

(d)

Figure 3. The $\{\boldsymbol{p}, \boldsymbol{p}$ ' $\}$ path-packets of the Fe-doped and undoped quinone switch with F(UP) decoration presented in sub-figures (a-b) and F(DOWN) in sub-figures (c-d) respectively, see the caption of Figure 2 for further details. Note, the extremely long $\{\boldsymbol{p}, \boldsymbol{p}$ '\} path-packets associated with the Fe-C BCPs in sub-figures (a) and (c) have been omitted for clarity here but are provided in the Supplementary Materials S4. 
Table 1. The lengths $\left\{\mathrm{H}^{*}, \mathrm{H}^{*}\right\},\left\{\mathrm{H}, \mathrm{H}^{\prime}\right\},\left\{\mathrm{H}_{\sigma}{ }^{*}, \mathrm{H}_{\sigma}{ }^{*}\right\},\left\{\mathrm{H}_{\sigma}, \mathrm{H}_{\sigma}{ }^{\prime}\right\}$ and BPL of the components of the bond-path framework set $\mathrm{B}=\{\boldsymbol{p}, \boldsymbol{q}, \boldsymbol{r}\}$ for the (r, TS, f) reverse minima, transition state and forward minima respectively for the N10-H13 $B C P$ of the F (UP and DOWN) decoration without and with the coordinated Fe atom.

\begin{tabular}{|c|c|c|}
\hline & $\mathbf{U P}$ & DOWN \\
\hline & $(\mathrm{r}, \mathrm{TS}, \mathrm{f})$ & $(\mathrm{r}, \mathrm{TS}, \mathrm{f})$ \\
\hline BCP & $\left\{\mathrm{H}^{*}, \mathrm{H}^{*}\right\}$ & $\left\{\mathrm{H}^{*}, \mathrm{H}^{*}\right\}$ \\
\hline N10-H13 & $\{(2.292,2.297),(2.876,2.938),(4.413,4.440)\}$ & $\{(2.327,2.322),(2.964,2.903),(4.663,4.636)\}$ \\
\hline N10-H13(Fe) & $\{(2.308,2.302),(2.706,2.649),(10.24,10.32)\}$ & $\{(2.303,2.309),(2.741,2.681),(13.92,14.00)\}$ \\
\hline Fe18-C3 & $\{(20.24,20.30),(28.95,29.06),(25.08,25.23)\}$ & $\{(19.18,19.10),(29.75,29.68),(31.29,31.37)\}$ \\
\hline Fe18-C6 & $\{(16.00,15.92),(25.47,25.55),(35.42,35.50)\}$ & $\{(17.26,17.32),(23.69,23.70),(26.17,26.18)\}$ \\
\hline BCP & $\left\{H_{,} H^{\prime}\right\}$ & $\left\{{\left.\mathrm{H}, \mathrm{H}^{\prime}\right\}}\right.$ \\
\hline N10-H13 & $\{(2.295,2.294),(2.919,2.902),(4.308,4.852)\}$ & $\{(2.325,2.324),(2.937,2.980),(4.556,5.147)\}$ \\
\hline N10-H13(Fe) & $\{(2.305,2.305),(2.675,2.682),(11.59,11.15)\}$ & $\{(2.306,2.306),(2.714,2.710),(15.28,15.59)\}$ \\
\hline Fe18-C3 & $\{(21.62,21.58),(30.99,30.88),(26.87,26.70)\}$ & $\{(20.30,20.35),(31.62,31.73),(33.63,33.45)\}$ \\
\hline Fe18-C6 & $\{(17.06,17.06),(27.38,27.40),(37.98,37.89)\}$ & $\{(18.47,18.41),(25.46,25.40),(28.04,28.11)\}$ \\
\hline$B C P$ & $\{\mathrm{BPL}\}$ & $\{\mathrm{BPL}\}$ \\
\hline N10-H13 & $\{(1.864),(2.727),(3.754)\}$ & $\{(1.863),(2.761),(3.811)\}$ \\
\hline N10-H13(Fe) & $\{(1.866),(2.530),(4.366)\}$ & $\{(1.868),(2.554),(4.424)\}$ \\
\hline Fe18-C3 & $\{(4.021),(4.022),(3.934)\}$ & $\{(4.032),(4.045),(3.975)\}$ \\
\hline Fe18-C6 & $\{(4.031),(4.040),(4.036)\}$ & $\{(4.009),(4.023),(4.021)\}$ \\
\hline$B C P$ & $\left\{\mathrm{H}_{\sigma}{ }^{*}, \mathrm{H}_{\sigma}{ }^{*}\right\}$ & $\left\{\mathrm{H}_{\sigma}{ }^{*}, \mathrm{H}_{\sigma}{ }^{*}\right\}$ \\
\hline N10-H13 & $\{(1.893,1.892),(2.743,2.743),(3.846,3.847)\}$ & $\{(1.893,1.894),(2.776,2.776),(3.911,3.911)\}$ \\
\hline N10-H13(Fe) & $\{(1.894,1.897),(2.541,2.541),(4.584,4.550)\}$ & $\{(1.896,1.899),(2.569,2.569),(4.655,4.622)\}$ \\
\hline Fe18-C3 & $\{(4.074,4.092),(4.083,4.094),(4.009,4.020)\}$ & $\{(4.086,4.074),(4.114,4.097),(4.049,4.033)\}$ \\
\hline Fe18-C6 & $\{(4.069,4.073),(4.098,4.090),(4.110,4.100)\}$ & $\{(4.063,4.077),(4.090,4.084),(4.094,4.092)\}$ \\
\hline BCP & $\left\{\mathrm{H}_{\sigma}, \mathrm{H}_{\sigma}{ }^{\prime}\right\}$ & $\left\{\mathrm{H}_{\sigma}, \mathrm{H}_{\sigma}{ }^{\prime}\right\}$ \\
\hline N10-H13 & $\{(1.894,1.892),(2.745,2.747),(3.950,3.760)\}$ & $\{(1.892,1.895),(2.778,2.780),(4.020,3.281)\}$ \\
\hline N10-H13(Fe) & $\{(1.894,1.897),(2.561,2.526),(4.470,4.706)\}$ & $\{(1.899,1.896),(2.562,2.585),(4.766,4.553)\}$ \\
\hline Fe18-C3 & $\{(4.168,4.181),(4.173,4.205),(4.122,4.709)\}$ & $\{(4.175,4.183),(4.222,4.203),(4.182,4.154)\}$ \\
\hline Fe18-C6 & $\{(4.222,4.196),(4.203,4.225),(4.209,4.229)\}$ & $\{(4.172,4.213),(4.222,4.195),(4.208,4.209)\}$ \\
\hline
\end{tabular}

The lengths of the conventional QTAIM bond-paths (BPL) $\boldsymbol{r}$ provide the minimal requirement for the condition of bonding and indicate a dependence on the position of the F-substituent and presence of the Fe atom, see Table 1. The lengths $\left\{\mathrm{H}^{*}, \mathrm{H}^{*}\right\},\left\{\mathrm{H}, \mathrm{H}^{\prime}\right\}$ of the $p$ - and $\boldsymbol{q}$-paths however, demonstrate larger differences due to the position of the F-substituent and presence of the Fe atom than the BPL, see Figures 23. This is seen in particular from the $q$-paths corresponding to the $\left\{\mathrm{H}^{*}, \mathrm{H}^{*}\right\}$ and $\left\{\mathrm{H}_{,} \mathrm{H}^{\prime}\right\}$ of the closed-shell Fe18--C3 $B C P$ and Fe18--C6 BCP of the forward (f) minimum for the Fe-doped compared with the undoped minimum, see the Supplementary Materials S3-S4. This is not the case for the corresponding $\left\{\mathrm{H}_{\sigma}{ }^{*}, \mathrm{H}_{\sigma}{ }^{*}\right\}$, $\left\{\mathrm{H}_{\sigma}, \mathrm{H}_{\sigma}\right\}$ lengths for the stress tensor, see Figures 4-5. 

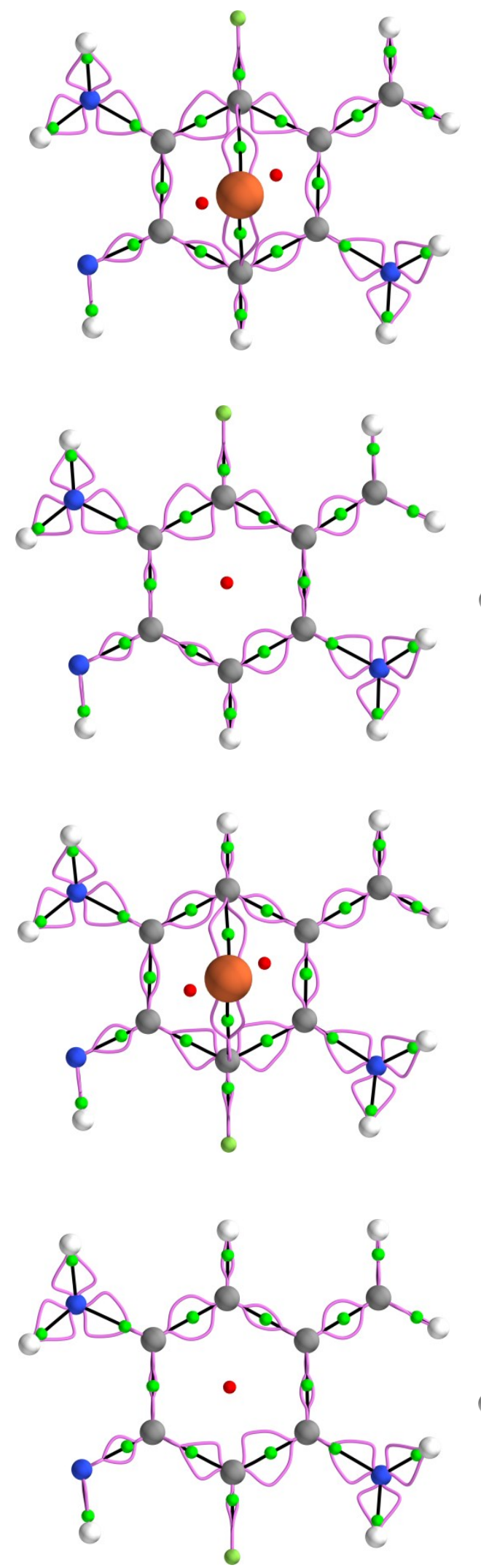

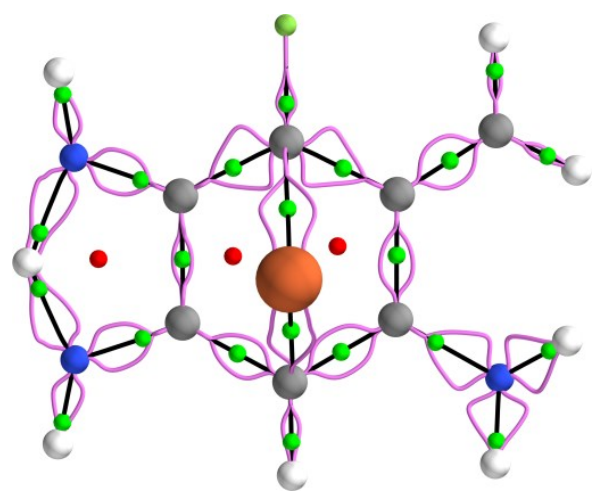

(a)

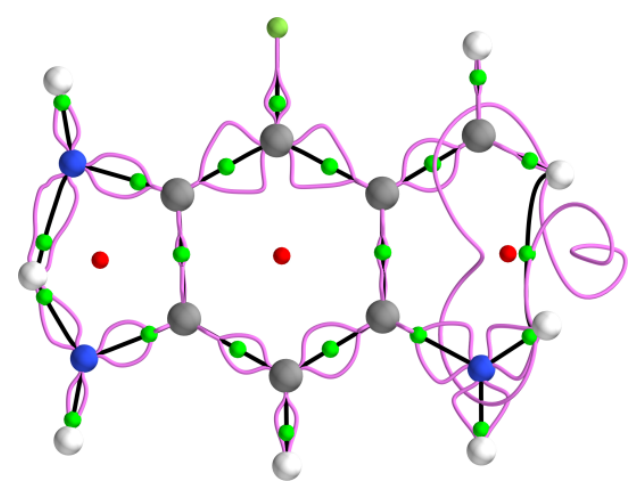

(b)

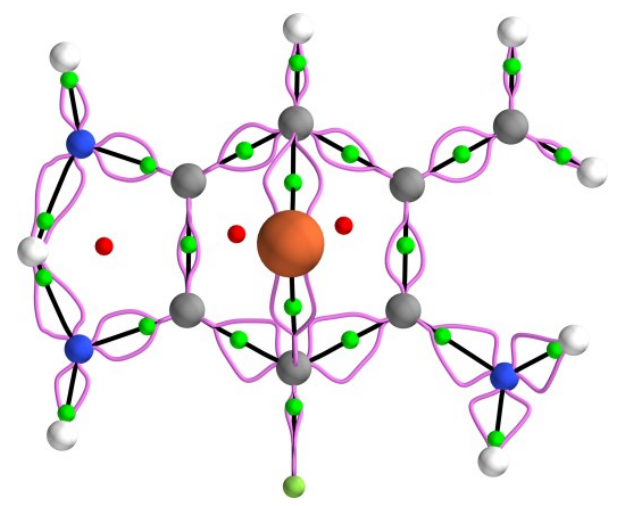

(c)

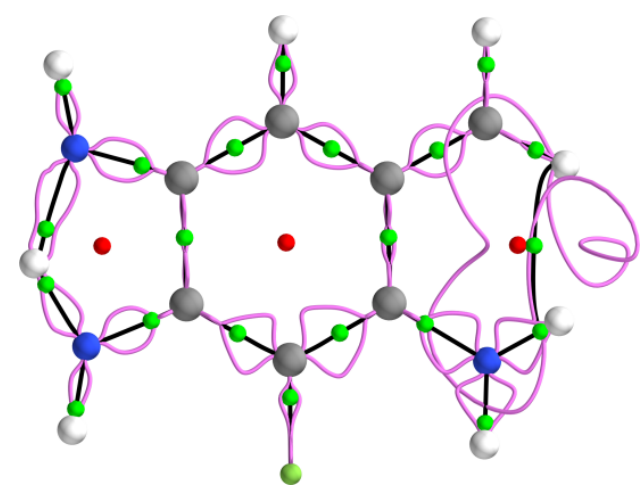

(d)
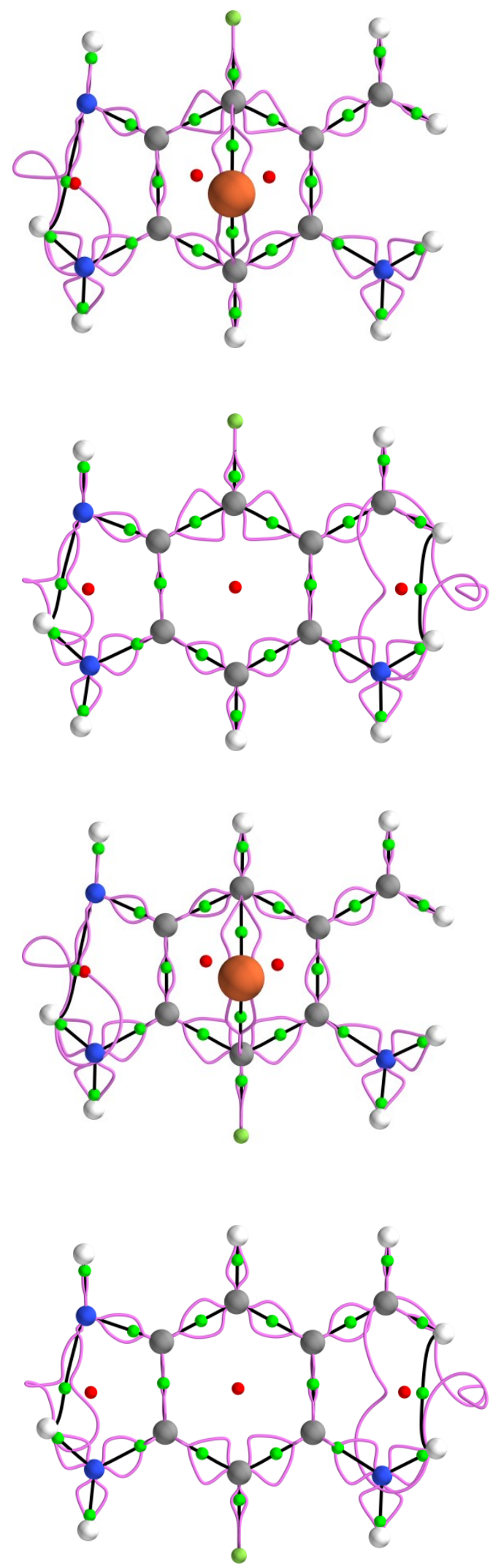

Figure 4. The $\left\{\boldsymbol{q}_{\sigma}, \boldsymbol{q}_{\sigma}\right.$ ' $\}$ path-packets presented with a magnification factor of $x 5$ for the Fe-doped and undoped quinone switch for the F(UP) decoration are presented in sub-figures (a-b) and F(DOWN) in sub-figures (c-d), see the caption of Figure 2 for further details. 

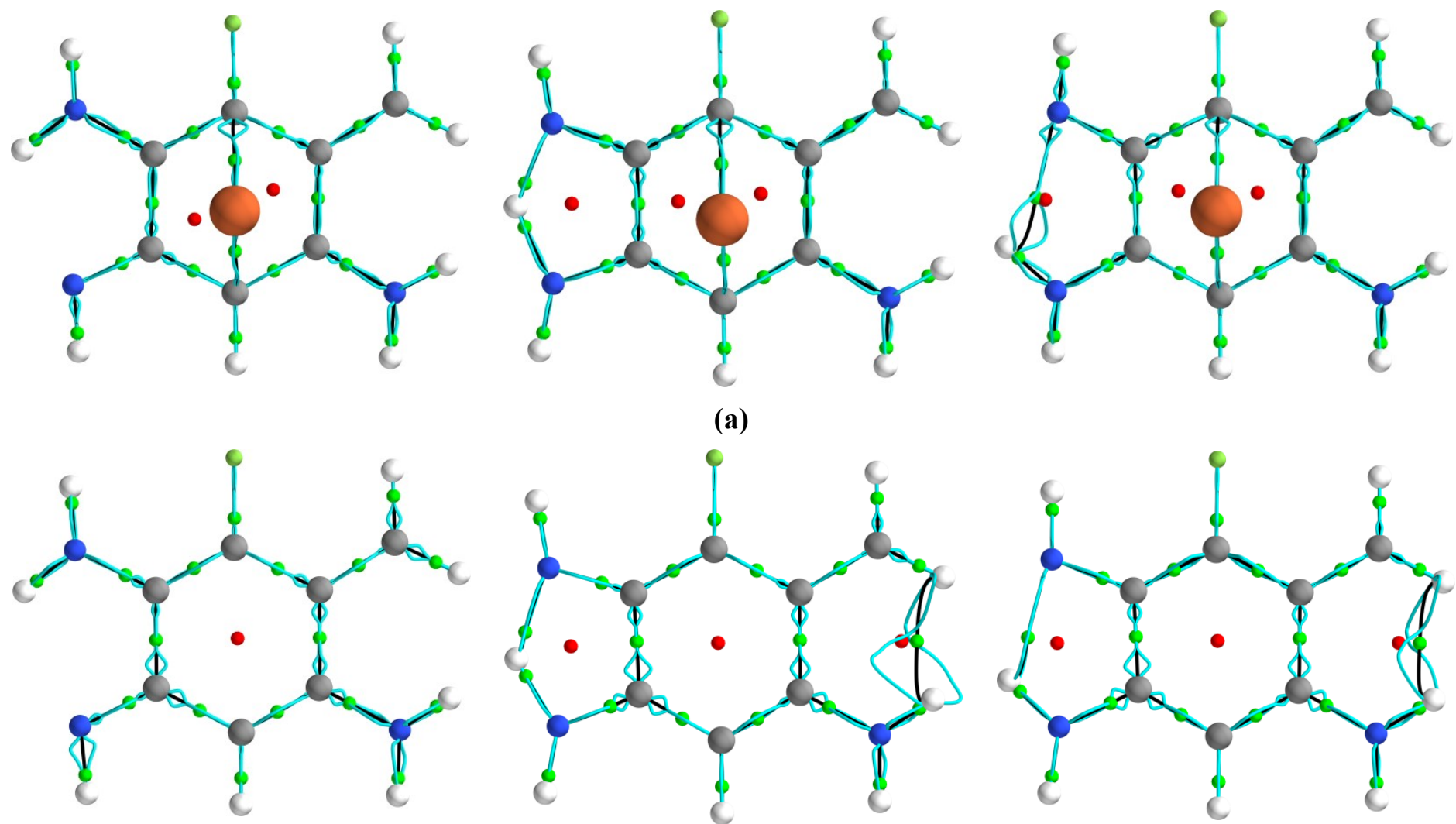

(b)
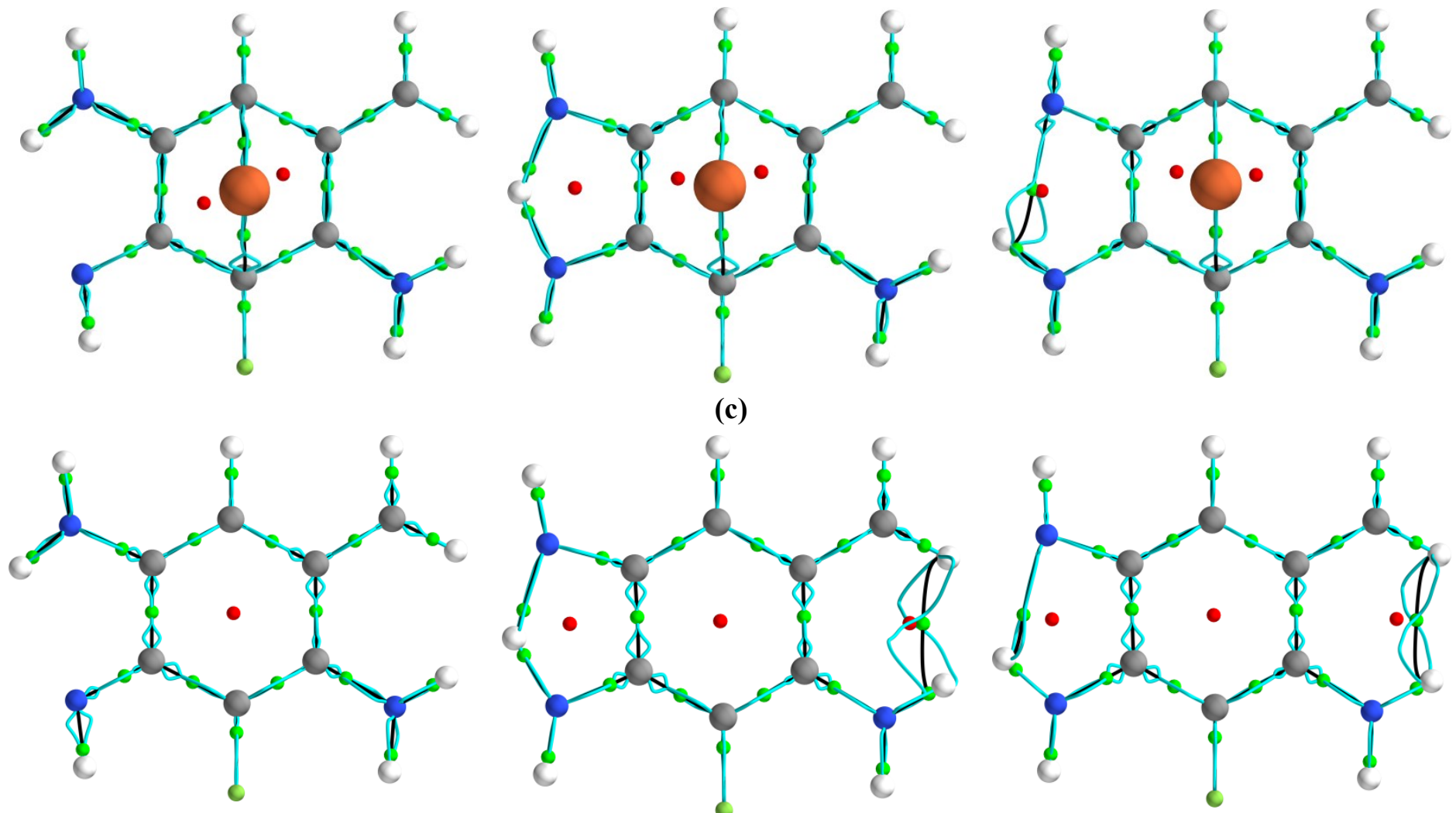

(d)

Figure 5. The $\left\{\boldsymbol{p}_{\sigma}, \boldsymbol{p}_{\sigma}\right.$ ' $\}$ path-packets magnified by a factor of 5 for the Fe-doped and undoped quinone switch with F(UP) decoration are presented in sub-figures (a-b) and F(DOWN) in sub-figures (c-d), see the caption of Figure $\mathbf{2}$ for further details. 
To summarize, the presence of metallic (i.e. $\left.\xi\left(\mathbf{r}_{\mathrm{b}}\right) \geq 1\right)$ N10--H13 BCPs was found to correlate with the relative energy $\Delta \mathrm{E}$ values demonstrating the major and minor effects of the addition of the Fe atom and UP or DOWN position of the F-substituent respectively. Greater stabilities from relative energy $\Delta \mathrm{E}$ values were determined by the greater persistence of the metallic N10--H13 BCPs along the forward direction of the IRC. This suggests that the $\mathrm{H} \mathrm{NCP}$ transfer tautomerization is facilitated by the presence of persistent metallic N10--H13 BCPs that were maximized in the Fe-doped switch with the F-substituent in the UP position. The metallicity $\xi\left(\mathbf{r}_{b}\right)$ is evidence that the presence of the $\mathrm{Fe}$ atom facilitates the $\mathrm{H} N C P$ transfer tautomerization by greater destabilization of the N10--H13 BCP bond-path that enables the easier motion of the H NCP. Further evidence of this greater destabilization was found from the shorter $B C P-R C P$ separation for the Fe-doped compared with undoped switch. This resulted in the $\left\{\boldsymbol{q}, \boldsymbol{q}^{\prime}\right\}$ and $\left\{\boldsymbol{q}_{\sigma}, \boldsymbol{q}_{\sigma}{ }_{\sigma}\right\}$ path-packets for the Fe-doped switch that correspond to the most preferred directions of electronic charge density accumulation, displaying much larger extents in the vicinity of the N10--H13 BCP than for the undoped switch. The larger extents of the $\{\boldsymbol{q}, \boldsymbol{q}$ ' $\}$ and $\left\{\boldsymbol{q}_{\sigma}, \boldsymbol{q}^{\prime}{ }_{\sigma}\right\}$ path-packets signify an easier passage of the $B C P$ and associated $\mathrm{H} N C P$ in the case of the Fe-doped compared with the undoped switch.

Differences between the metallicity $\xi\left(\mathbf{r}_{\mathrm{b}}\right)$ values for the UP and DOWN F-substituent positions and the lengths $\left\{\mathrm{H}^{*}, \mathrm{H}^{*}\right\},\left\{\mathrm{H}, \mathrm{H}^{\prime}\right\},\left\{\mathrm{H}_{\sigma}{ }^{*}, \mathrm{H}_{\sigma}{ }^{*}\right\}$, and $\left\{\mathrm{H}_{\sigma}, \mathrm{H}_{\sigma}{ }^{\prime}\right\}$ of the $\boldsymbol{p}-, \boldsymbol{q}-, \boldsymbol{p}_{\sigma^{-}}$, and $\boldsymbol{q}_{\sigma^{-}}$respectively, indicate that the addition of the Fe atom displays the positional effect of the F-substituent more sensitively than the BPL.

The stress tensor $\left\{\boldsymbol{p}_{\sigma}, \boldsymbol{q}_{\sigma}\right\}$ and the original QTAIM $\{\boldsymbol{p}, \boldsymbol{q}\}$ path-packets both display the least and most preferred directions of electronic charge density accumulation compared with the minimal definition of bonding $r$. The main advantage of using the $B_{\sigma}$ is the tractable $\left\{\mathrm{H}_{\sigma}, \mathrm{H}_{\sigma}{ }^{\prime}\right\}$ lengths, of the $\boldsymbol{q}_{\sigma}$-paths that are readily visualized, e.g. associated with the closed-shell Fe--C BCPs and closed-shell $\mathrm{H}---\mathrm{H} B C P$ bond-paths. Conversely, the corresponding $\left\{\mathrm{H}_{,} \mathrm{H}^{\prime}\right\}$ lengths of the $\boldsymbol{q}$-paths for B from QTAIM are not as readily visualized.

\section{Acknowledgements}

The National Natural Science Foundation of China is acknowledged, project approval number: 21673071. The One Hundred Talents Foundation of Hunan Province is also gratefully acknowledged for the support of S.J. and S.R.K. The Royal Society is thanked by S.J., S.R.K, T.X, T.v.M and H.F. for support through an International Exchanges grant. We thank EaStCHEM for computational support via the EaStCHEM Research Computing Facility. 


\section{References}

1. Fruchtl, H. \& van Mourik, T. A Quinone Based Single-Molecule Switch as Building Block for Molecular Electronics. arXiv:1801.01001 [physics] (2018).

2. Zhang, J. L., Zhong, J. Q., Lin, J. D., Hu, W. P., Wu, K., Xu, G. Q., Wee, A. T. S. \& Chen, W. Towards single molecule switches. Chem. Soc. Rev. 44, 2998-3022 (2015).

3. Komoto, Y., Fujii, S., Iwane, M. \& Kiguchi, M. Single-molecule junctions for molecular electronics. J. Mater. Chem. $C 4,8842-8858$ (2016).

4. Ayers, P. W. \& Jenkins, S. Bond metallicity measures. Computational and Theoretical Chemistry 1053, 112-122 (2015).

5. Jenkins, S., Maza, J. R., Xu, T., Jiajun, D. \& Kirk, S. R. Biphenyl: A stress tensor and vector-based perspective explored within the quantum theory of atoms in molecules. International Journal of Quantum Chemistry 115, 1678-1690 (2015).

6. Jiajun, D., Maza, J. R., Xu, Y., Xu, T., Momen, R., Kirk, S. R. \& Jenkins, S. A stress tensor and QTAIM perspective on the substituent effects of biphenyl subjected to torsion. Journal of Computational Chemistry 37, 2508-2517 (2016).

7. Guo, H., Morales-Bayuelo, A., Xu, T., Momen, R., Wang, L. Yang, P., Kirk, S. R. \& Jenkins, S. Distinguishing and quantifying the torquoselectivity in competitive ring- opening reactions using the stress tensor and QTAIM. Journal of Computational Chemistry 37, 2722-2733 (2016).

8. Xu, T., Wang, L. Yang, P., Mourik, T., Früchtl, H., Kirk, S. R. \& Jenkins, S. Quinone-based switches for candidate building blocks of molecular junctions with QTAIM and the stress tensor. International Journal of Quantum Chemistry 118, e25676 (2018).

9. Bader, R. F. W. Atoms in Molecules: A Quantum Theory. (Oxford University Press, USA, 1994).

10. Tian, T., Xu, T., Kirk, S. R., Filatov, M. \& Jenkins, S. Next-generation quantum theory of atoms in molecules for the ground and excited state of the ring-opening of cyclohexadiene. International Journal of Quantum Chemistry 0, e25862

11. Bader, R. F. W. A Bond Path: A Universal Indicator of Bonded Interactions. J. Phys. Chem. A 102, 7314-7323 (1998).

12. Bader, R. F. W. Bond Paths Are Not Chemical Bonds. J. Phys. Chem. A 113, 10391-10396 (2009).

13. Kraka, E. Description of chemical reactions in terms of the properties of the electron density. Journal of Molecular Structure: THEOCHEM 255, 189-206 (1992).

14. Jenkins, S., Blancafort, L., Kirk, S. R. \& Bearpark, M. J. The response of the electronic structure to electronic excitation and double bond torsion in fulvene: a combined QTAIM, stress tensor and MO perspective. Phys. Chem. Chem. Phys. 16, 7115-7126 (2014).

15. Jenkins, S. Direct space representation of metallicity and structural stability in SiO solids. J. Phys.: Condens. Matter 14, 10251-10263 (2002).

16. Jenkins, S., Ayers, P. W., Kirk, S. R., Mori-Sánchez, P. \& Martín Pendás, A. Bond metallicity of materials from real space charge density distributions. Chemical Physics Letters 471, 174-177 (2009).

17. M. J. Frisch, G. W. Trucks, H. B. Schlegel, G. E. Scuseria, M. A. Robb, J. R. Cheeseman, G. Scalmani, V. Barone, G. A. Petersson, H. Nakatsuji, X. Li, M. Caricato, A. Marenich, J. Bloino, B. G. Janesko, R. Gomperts, B. Mennucci, H. P. Hratchian, J. V. Ortiz, A. F. Izmaylov, J. L. Sonnenberg, D. Williams-Young, F. Ding, F. Lipparini, F. Egidi, J. Goings, B. Peng, A. Petrone, T. Henderson, D. Ranasinghe, V. G. Zakrzewski, J. Gao, N. Rega, G. Zheng, W. Liang, M. Hada, M. Ehara, K. Toyota, R. Fukuda, J. Hasegawa, M. Ishida, T. Nakajima, Y. Honda, O. Kitao, H. Nakai, T. Vreven, K. Throssell, J. A. Montgomery, Jr., J. E. Peralta, F. Ogliaro, M. Bearpark, J. J. Heyd, E. Brothers, K. N. Kudin, V. N. Staroverov, T. Keith, R. Kobayashi, J. Normand, K. Raghavachari, A. Rendell, J. C. Burant, S. S. Iyengar, J. Tomasi, M. Cossi, J. M. Millam, M. Klene, C. Adamo, R. Cammi, J. W. Ochterski, R. L. Martin, K. Morokuma, O. Farkas, J. B. Foresman, and D. J. Fox. Gaussian 09, Revision D.01. (Gaussian, Inc., 2009).

18. Adamo, C. \& Barone, V. Toward reliable density functional methods without adjustable parameters: The PBE0 model. The Journal of Chemical Physics 110, 6158-6170 (1999).

19. Dunning, T. H. Gaussian basis sets for use in correlated molecular calculations. I. The atoms boron through neon and hydrogen. The Journal of Chemical Physics 90, 1007-1023 (1989).

20. Grimme, S., Antony, J., Ehrlich, S. \& Krieg, H. A consistent and accurate ab initio parametrization of density functional dispersion correction (DFT-D) for the 94 elements H-Pu. The Journal of Chemical Physics 132, 154104 (2010).

21. Grimme, S., Ehrlich, S. \& Goerigk, L. Effect of the damping function in dispersion corrected density functional theory. J. Comput. Chem. 32, 1456-1465 (2011).

22. Keith, T. A. AIMAll, Revision 17.01.25. (TK Gristmill Software, 2017). 